\title{
Taurolock Effectively Reduces Tunneled Dialysis Catheter-Related Infection and Catheter Exchange Rates among Hemodialysis Patients
}

\author{
Hassan Al Malki*, Muftah Othman, Baha Osman, Awad Rashid, Omar Fituri, \\ Muhammad Asim \\ Hamad General Hospital, Hamad Medical Corporation, Doha, Qatar \\ Email: "halmalki1@hamad.qa
}

Received 30 March 2016; accepted 11 June 2016; published 14 June 2016

Copyright (C) 2016 by authors and Scientific Research Publishing Inc. This work is licensed under the Creative Commons Attribution International License (CC BY). http://creativecommons.org/licenses/by/4.0/

(c) (i) Open Access

\begin{abstract}
Aims: Catheter-related infection, which is one of the major side effects of the use of dialysis catheters, leads to increases in hospitalization, morbidity and mortality. Antibiotic lock is an option for reducing the incidence of these infections, but there are concerns regarding antibiotic resistance. A prior study demonstrated that Taurolock (a taurolidine lock) may reduce the rate of catheter-related infection. Methods and Material: This investigation was a prospective before-and-after study. During period one, patients continued to use a heparin lock $(5000$ units $/ \mathrm{ml})$ for 6 months. During period two, they were shifted to Taurolock (a solution of $1.35 \%$ taurolidine, $4 \%$ citrate, and 500 units $/ \mathrm{ml}$ of heparin) for 6 months. The primary outcome was the incidence of tunneled catheter-related infection and/or catheter exchange, and the secondary outcomes were the effects of Taurolock on catheter flow rate, dialysis adequacy, and catheter malfunction rate. Results: A total of 49 patients were included in this study. During period one, the primary outcome occurred 17 times, with an incidence rate of 86.8 per 100 patient-years; during period two, the primary outcome occurred 7 times, with an incidence rate of 32.6 per 100 patient-years (incidence rate ratio: $2.65,95 \%$ confidence interval $(\mathrm{CI}): 1.05-7.6, \mathrm{P}=\mathbf{0 . 0 2 3}$ ). There was no significant difference between the two periods with respect to mean catheter blood flow rate $(P=0.29)$. During period one, thrombolytic therapy (TPA) lock was indicated on 19 occasions, with an incidence rate of 97 per 100 patient-years; during period two, TPA lock was indicated on 53 occasions, with an incidence rate of 247.5 per 100 patient-years (incidence rate ratio: $0.3,95 \%$ CI: $0.21-0.67, \mathrm{P}=$ 0.0003). Conclusions: We demonstrated that Taurolock usage is safe and effective for the prevention of dialysis catheter-related infection and/or catheter exchange.
\end{abstract}

\footnotetext{
${ }^{*}$ Corresponding author.
} 


\section{Keywords}

\section{Catheter, Hemodialysis, Infection, Prevention, Lock}

\section{Introduction}

Hemodialysis (HD), which is the most common type of renal replacement therapy, requires vascular access to be obtained (via a temporary catheter, tunneled catheter, fistula, or graft) [1]-[3].

Central venous catheters are used to provide blood access for HD patients, although these catheters are generally only reluctantly employed due to safety concerns and associated frequent complications (mainly line infection). Tunneled catheters are often used for relatively lengthy time periods, and catheter-related bacteremia is a major source of morbidity, hospitalization, and economic expense among catheter-dependent patients. Most episodes of catheter-related bacteremia are relatively uncomplicated; however, $10 \%$ to $20 \%$ of these episodes are complicated by metastatic infection, with manifestations that include endocarditis, septic arthritis, and epidural abscess. The risk of catheter-related bacteremia is proportional to the duration of central venous catheter use; in particular, this type of bacteremia occurs in 35\% of patients within 3 months and $48 \%$ of patients within 6 months and exhibits an incidence rate of 0.9 to 2.0 episodes per catheter-year [4]-[7].

Antibiotic lock has been proposed as a method for preventing catheter-related infection, although the main concern associated with this treatment is the development of antibiotic resistance in dialysis patients. Taurolidine, which has been used in Europe since the 1970s as an antimicrobial lavage for treating peritonitis and various types of wounds, has been used as a locking solution in combination with sodium citrate, which acts as an anticoagulant. Research has indicated that taurolidine is effective against a broad range of Gram-negative and Gram-positive bacteria and many types of fungi. In addition to being effective, taurolidine appears to be safe, and its use has not been associated with the side effects that have been reported for certain antibiotic lock solutions. In fact, small studies have indicated that taurolidine reduces the rate of catheter-related infection with no side effects [8]-[10].

In this investigation, we examined the use of a taurolidine lock solution (Taurolock) in patients with tunneled catheters; in particular, the rates of catheter-related infection and/or required catheter exchange were compared before and after the introduction of this lock solution among patients treated in our unit at Hamad Medical Corporation (HMC), Doha, Qatar.

This investigation sought to compare the incidences of catheter-related infections and/or catheter exchange before and after the introduction of Taurolock in our HD unit at HMC and to determine the effects of Taurolock on catheter flow rate, HD adequacy, and thrombosis rate.

\section{Subjects \& Methods}

This study was performed at two satellite centers affiliated with HMC. All catheters were placed at our main hospital (Hamad General Hospital) by vascular surgeons. All catheters were jugular and were placed under fluoroscopy with prophylactic antibiotic coverage.

Patients were recruited from December 2009 to June 2011.

This investigation was a prospective study that compared the efficacies of Taurolock (a new lock solution at HMC) and heparin (a lock solution that has been in use for an extended period) with respect to reducing catheter-related infection and/or catheter exchange.

After recruitment, patients continued to use a heparin lock (5000 units/ml) for 6 months (period one). Subsequently, they were shifted to Taurolock (a solution of $1.35 \%$ taurolidine, $4 \%$ citrate, and 500 units $/ \mathrm{ml}$ of heparin, Tauropharm, Waldbüttelbrunn, Germany) for 6 months (period two).

Inclusion criteria were adult patients who are dialyzed via a tunneled catheter. All patients who had both treatment modalities were included in the study.

Exclusion criteria were patients who had a catheter-related infection within 3 months prior to the start of the study, patients who are being treated with immunosuppressants (for any reason), patients with malignancies, and patients who are receiving chemotherapy.

The primary outcome of this study was the incidence of tunneled catheter-related infection and/or catheter 
exchange. The incidences of these events before and after the introduction of Taurolock were compared for our dialysis patients. The secondary outcomes of this study were catheter flow rate, dialysis adequacy, and catheter thrombosis/malfunction rate. These outcomes were compared for our dialysis patients before and after the introduction of Taurolock. Infection was diagnosed if the patient developed a fever and had positive blood cultures of the same organism from both the central and peripheral lines in the absence of any other detected sources of infection. These infections were treated in accordance with our local protocol, which consisted of the administration of empirical antibiotics, including vancomycin and amikacin, and subsequent changes in the antibiotic regimen based on culture sensitivity. In all cases of tunneled catheter malfunction, thrombolytic lock was attempted in accordance with our local protocol, which consisted of three treatments with tissue plasminogen activator $(1 \mathrm{mg} / \mathrm{ml})$ followed by the performance of catheter exchange by our local surgeon.

This study was done according the Helsinki declaration, and the study protocol had been approved by the local institute's Committee on Human Research (our local ethical body). Informed consent has been obtained from all patients included in the study.

Data were tabulated using Microsoft Excel, and the Pearson chi-square test was used to assess these data. Differences in mean blood flow and dialysis clearance rates were compared using t-tests. Countable outcomes were measured as rates of occurrence/100 patient-years, with $\mathrm{P}<0.05$ regarded as statistically significant. Statistical analysis was performed using SPSS 17.0, a commercial statistical package for the social sciences.

\section{Results}

During the study period, we treated 136 patients with tunneled catheters, 49 of whom were treated with both heparin and Taurolock (36\% of all patients) [Table 1].

\subsection{Primary Outcome}

The incidence of the primary outcome (tunneled catheter-related infection and/or catheter exchange) was 17 events during period one, with an incidence rate of 86.8 per 100 patient-years, and 7 events during period two, with an incidence rate of 32.6 per 100 patient-years (incidence rate ratio: 2.65, 95\% CI: 1.05 - 7.6, P = 0.023).

\subsection{Secondary Outcomes}

There were 12 tunneled catheter-related infections during the study: 8 infections (66.6\%) occurred during period one, with an incidence rate of 40.8 per 100 patient-years, and 4 infections (33.4\%) occurred during period two, with an incidence rate of 18.6 per 100 patient-years. Thus, the incidence rate for tunneled catheter-related infection was 22.1 less per 100 patient-years during period two than during period one (incidence rate ratio: 2.1, 95\% CI: 0.58 - 9.9, $\mathrm{P}=0.1$ ).

During period one, 9 catheters required exchange, with an incidence rate of 45.9 per 100 patient-years; during period two, only 3 catheters required exchange, with an incidence rate of 14 per 100 patient-years. The incidence rate of catheter exchange was 31.9 per 100 patient-years less during period two than during period one (incidence rate ratio: $3.2,95 \% \mathrm{CI}: 0.8-18.8, \mathrm{P}=0.05$ ).

There was no significant difference between the two periods with respect to mean catheter blood flow rate, which was $249.2 \mathrm{ml} / \mathrm{min}$ during period one and $245.6 \mathrm{ml} / \mathrm{min}$ during period two $(\mathrm{P}=0.29)$.

Table 1. Basic demographics.

\begin{tabular}{cc}
\hline Male & Number (\%) \\
Female & $29(59.1)$ \\
Diabetes Mellitus & $20(40.9)$ \\
Hypertension & $29(59.1)$ \\
Glomerulonephritis & $4(16.4)$ \\
Coronary Artery Disease & $21(8.2)$ \\
Peripheral Vascular disease & $7(14.8)$ \\
Chronic Liver Disease & $2(4.1)$ \\
\hline
\end{tabular}


Similarly, the dialysis clearance results (as measured by urea reduction ratio (URR)) were comparable between the two periods: during periods one and two, the mean URRs were $57.7 \%$ and $57.4 \%$, respectively (P = $0.8)$.

Thrombolytic (TPA) locks were required by fewer patients during period one than during period two. During period one, TPA locks were required 19 times, with an incidence rate of 97 per 100 patient-years; during period two, TPA locks were required 53 times, with an incidence rate of 247.5 per 100 patient-years (incidence rate ratio: 0.3 , 95\% CI: $0.21-0.67, \mathrm{P}=0.0003)$.

We found no differences between the two periods with respect to patterns of infection or bacterial antibiotic sensitivities $(\mathrm{P}=0.25)$. Furthermore, there was no observed development of new antibiotic-resistant organisms during either period.

\section{Discussion}

The identification of a lock solution for dialysis catheters that can simultaneously reduce infection rates without affecting catheter function has always been greatly desired by clinicians [11].

In this prospective study, we compared the rates of catheter-related infection and/or required catheter exchange during two study periods. During the first study period, we used heparin, which is the standard anticoagulant administered in most dialysis units around the world. During the second study period, we used Taurolock solution, which has become the standard lock solution at our institution.

Our data revealed a significant reduction in the rate of catheter-related infection and/or catheter exchange from period one to period two; in particular, this rate decreased from 86.8 to 32.6 per 100 patient-years, with a significant $\mathrm{P}$ value of 0.023 . The combined outcome of catheter-related infection and/or catheter exchange was used because both of these events are highly important with respect to long-term morbidity and mortality among HD patients [12] [13]. To the best of our knowledge, this investigation is the only study to date to examine the combination of these two hard end points as the primary outcome.

The rate of catheter-related infection was impressively reduced from 40.8 episodes to 18.6 episodes per 100 patient-years from period one to period two; this result regarding reduced catheter-related infections was consistent with previous findings [14]-[16].

In contrast to prior studies indicating that the use of Taurolock was associated with a higher rate of catheter exchange, our data revealed that catheter exchange was required less frequently during period two than during period one, with 14 exchanges per 100 patient-years during period two and 45 exchanges per 100 patient-years during period one $(\mathrm{P}=0.05)$. Therefore, the greatest benefit of Taurolock may be a reduction in the rate of dialysis catheter exchange [17] [18].

No previous studies have reported data regarding the effects of Taurolock on blood flow and dialysis clearance rates. Our results strongly indicated that Taurolock was comparable to heparin with respect to blood flow rate (245.6 versus 249.2, $\mathrm{P}=0.29$ ). Similarly, dialysis clearance rates were comparable during the Taurolock and heparin phases (57.4 versus $57.7, \mathrm{P}=0.8$ ).

One major reported disadvantage of using Taurolock is the increased need for TPA to maintain catheter patency [19]-[21]. Our results were consistent with these prior findings; in particular, in our study, the rate of thrombolytic lock was 247 events per 100 patient-years during the Taurolock phase but only 97 events per 100 patient-years during the heparin phase. However, one reassuring consideration was that TPA was successful in most cases. During the Taurolock phase, three patients were very reluctant to undergo catheter exchange, and all three of these patients required more than six thrombolytic locks to maintain catheter patency and allow them to continue receiving their required dialysis; this phenomenon may explain the high rate of thrombolytic lock during the Taurolock phase.

This study had several limitations. First, this investigation was not a randomized controlled study, which would have been a better methodology than our study design for comparing the two lock solutions. Our center's policy was to shift all patients to Taurolock; therefore, randomization was not an option. Second, only 6 months of follow up were conducted; thus, we could not examine the long-term outcomes of treatment with the new lock solution. Finally, this study had a small sample size, which may affect the generalizability of our results to actual clinical practice.

\section{Conclusion}

In conclusion, we demonstrated that Taurolock usage is safe and effective for the prevention of dialysis cathe- 
ter-related infection and/or catheter exchange. The findings that the use of Taurolock instead of heparin did not affect catheter patency or dialysis clearance were reassuring. No development of antibiotic resistance was observed, and no complications with the new lock solution were reported.

\section{References}

[1] Andrew, S. and Coresh, J. (2002) K/DOQI Clinical Practice Guidelines for Chronic Kidney Disease: Evaluation, Classification, and Stratification. American Journal of Kidney Diseases, 39, S1-S266.

[2] Levey, A.S., Eckardt, K.U., Tsukamoto, Y., et al. (2005) Definition and Classification of Chronic Kidney Disease: A Position Statement from Kidney Disease: Improving Global Outcomes (KDIGO). Kidney International, 67, 2089-2100. http://dx.doi.org/10.1111/j.1523-1755.2005.00365.x

[3] Gilmore, J. (2006) K/DOQI Clinical Practice Guidelines and Clinical Practice Recommendations 2006 Updates. Nephrology Nursing Journal, 33, 487-488.

[4] Lee, T., Barker, J. and Allon, M. (2005) Tunneled Catheters in Hemodialysis Patients: Reasons and Subsequent Outcomes. American Journal of Kidney Diseases, 46, 501-508. http://dx.doi.org/10.1053/j.ajkd.2005.05.024

[5] Danese, M.D., Griffiths, R.I. and Dylan, M. (2006) Mortality Differences among Organisms Causing Septicemia in Hemodialysis Patients. Hemodialysis International, 10, 56-62. http://dx.doi.org/10.1111/j.1542-4758.2006.01175.x

[6] Nissenson, A.R., Dylan, M.L. and Griffiths, R.I. (2005) .Clinical and Economic Outcomes of Staphylococcus aureus Septicemia in ESRD Patients Receiving Hemodialysis. American Journal of Kidney Diseases, 46, 301-308. http://dx.doi.org/10.1053/j.ajkd.2005.04.019

[7] Abbas, S.A., Haloob, I.A. and Taylor, S.L. (2009) Effect of Antimicrobial Locks for Tunneled Hemodialysis Catheters on Bloodstream Infection and Bacterial Resistance: A Quality Improvement Report. American Journal of Kidney Diseases, 53, 492-502. http://dx.doi.org/10.1053/j.ajkd.2008.09.019

[8] Bleyer, A.J. (2007) Use of Antimicrobial Catheter Lock Solutions to Prevent Catheter-Related Bacteremia. Clinical Journal of the American Society of Nephrology, 2, 1073-1078. http://dx.doi.org/10.2215/CJN.00290107

[9] Yahav, D., Rozen-Zvi, B. and Gafter-Gvili, A. (2008) Antimicrobial Lock Solutions for the Prevention of Infections Associated with Intravascular Catheters in Patients Undergoing Hemodialysis: Systematic Review and Meta-Analysis of Randomized, Controlled Trials. Clinical Infectious Diseases, 4, 83-93. http://dx.doi.org/10.1086/588667

[10] Shah, C.B., Mittelman, M.W. and Costerton, J.W. (2002) Antimicrobial Activity of a Novel Catheter Lock Solution. Antimicrobial Agents and Chemotherapy, 46, 1674-1679. http://dx.doi.org/10.1128/AAC.46.6.1674-1679.2002

[11] Allon, M. (2008) Prophylaxis against Dialysis Catheter-Related Bacteremia: A Glimmer of Hope. American Journal of Kidney Diseases, 51, 165-168. http://dx.doi.org/10.1053/j.ajkd.2007.12.003

[12] Ridão Curty, N.F., da Silva Martins, L.F. and Sanches Ito, C.A. (2014) Morbimortality Study of Infection in Patients Undergoing Different Types of Dialysis in a Renal Replacement Therapy Center. Brazilian Journal of Infectious Diseases, 18, 281-286. http://dx.doi.org/10.1016/j.bjid.2013.08.007

[13] Ravani, P., Gillespie, B.W. and Quinn, R.R. (2013) Temporal Risk Profile for Infectious and Noninfectious Complications of Hemodialysis Access. Journal of the American Society of Nephrology, 24, 1668-1677. http://dx.doi.org/10.1681/ASN.2012121234

[14] Betjes, M.G. and van Agteren, M. (2004) Prevention of Dialysis Catheter-Related Sepsis with Acitrate-Taurolidine Containing Lock Solution. Nephrology Dialysis Transplantation, 19, 1546-1551. http://dx.doi.org/10.1093/ndt/gfh014

[15] Murray, E.C., Deighan, C. and Geddes, C. (2014) Taurolidine-Citrate-Heparin Catheter Lock Solution Reduces Staphylococcal Bacteraemia Rates in Haemodialysis Patients. QJM, 107, 995-1000. http://dx.doi.org/10.1093/qjmed/hcu128

[16] Taylor, C., Cahill, J. and Gerrish, M, (2008) A New Hemodialysis Catheter-Locking Agent Reduces Infections in Hemodialysis Patients. Journal of Renal Care, 34, 116-120. http://dx.doi.org/10.1111/j.1755-6686.2008.00027.x

[17] Solomon, L.R., Cheesbrough, J.S. and Ebah, L. (2010) A Randomized Double-Blind Controlled Trial of TaurolidineCitrate Catheter Locks for the Prevention of Bacteremia in Patients Treated with Hemodialysis. American Journal of Kidney Diseases, 55, 1060-1068. http://dx.doi.org/10.1053/j.ajkd.2009.11.025

[18] Liu, H., Liu, H. and Deng, J. (2014) Preventing Catheter-Related Bacteremia with Taurolidine-Citrate Catheter Locks: A Systematic Review and Meta-Analysis. Blood Purification, 37, 179-187. http://dx.doi.org/10.1159/000360271

[19] Fontseré, N., Cardozo, C. and Donate, J. (2014) Tunneled Catheters with Taurolidine-Citrate-Heparin Lock Solution Significantly Improve the Inflammatory Profile of Hemodialysis Patients. Antimicrobial Agents and Chemotherapy. Antimicrobial Agents and Chemotherapy, 58, 4180-4184. http://dx.doi.org/10.1128/AAC.02421-14 
[20] Allon, M. (2003) Prophylaxis against Dialysis Catheter-Related Bacteremia with a Novel Antimicrobial Lock Solution. Clinical Infectious Diseases, 36, 1539-1544. http://dx.doi.org/10.1086/375234

[21] Buturovic, J., Ponikva, R. and Kandus, A. (1998) Filling Hemodialysis Catheters in the Interdialytic Period. Heparin versus Citrate versus Polygeline: A Prospective Randomised Study. Artificial Organs, 22, 945-947.

http://dx.doi.org/10.1046/j.1525-1594.1998.06268.x 